Michael MEYER, Dortmund

\title{
Begriffsbildung durch Entdecken und Begründen
}

Am Beispiel des Begriffs „arithmetischer Mittelwert“ wird eine allgemeine Strategie erläutert, wie Lernende dazu angeregt werden können, einen mathematischen Begriff durch Prozesse des Entdeckens und Begründens zu bilden.

\section{Einleitung}

In der wissenschaftlichen Diskussion wird der Begriff „Begriff“ auf verschiedene Arten diskutiert. Zunächst soll zwischen dem Wort und der damit verbundenen Bedeutung unterschieden werden, so dass die Bezeichnung „Begriff“ beides umfasst. Die Bedeutung eines Begriffs wird zumeist nicht auf seine Definition beschränkt:

Einen „mathematischen Begriff zu ,besitzen’, erfordert, mehr Beziehungen zu kennen und mehr über den Umgang mit diesem Begriff zu wissen, als in der Definition ausgedrückt wird. [...] Beweise sind ein vorzügliches Mittel dazu, die innere Struktur von Begriffen zu explizieren sowie Begriffe miteinander zu vernetzen und damit den Bedeutungsgehalt von Begriffen zu entwickeln.“ (Fischer und Malle 1985, S. 189f)

In den mathematikdidaktischen Lehrwerken zur Begriffsbildung im Unterricht werden verschiedene Wege beschrieben, wie mathematische Begriffe den Lernenden nahegelegt werden können. Beispielweise unterscheidet Winter (1983) sechs verschiedene Arten der Begriffsbestimmung. Vollrath (2001) beschreibt, wie die Einführung mathematischer Begriffe in Problemkontexten geschehen kann. Entdeckungsprozesse werden von beiden Autoren hinsichtlich des sukzessiven Abstrahierens von Eigenschaften von konkreten Objekten oder Operationen, die der Experte als Instanziierungen des Begriffs auffasst, beschrieben. Der hier verwendete Begriff des „Entdeckens“ ist jedoch enger gefasst und beschreibt solche Prozesse, bei denen abduktiv schließend eine neue Erkenntnis im Sinne einer allgemeinen Aussage gewonnen wird. Der Mathematiker Rényi (1976, S. 28) nutzt zu Beschreibung des Lernens neuer Begriffe die Metapher eines Schiffsbaus und der anschließenden Reise mit diesem Schiff: Der Begriff wird zunächst eingeführt (das Schiff gebaut), etwa qua Definition, bevor er verwendet werden kann, um mathematische Zusammenhänge zu entdecken (mit dem fertigen Schiff neue Gestade entdecken) und als Sätze zu formulieren. Insofern wird nach Rényi ein Begriff nicht entdeckt, sondern gebildet (resp. „erfunden“, Götz und Siller 2010), bevor ein Satz entdeckt werden kann. 
In diesem Beitrag soll die Möglichkeit skizziert werden, wie umgekehrt mathematische Begriffe durch das Entdecken und Begründen mathematischer Sätze gebildet werden können. Diese Möglichkeit wird vereinzelt bereits in neueren Lehrwerken zum Mathematikunterricht genutzt und soll hier in ihrer allgemeinen Struktur am Beispiel des Mittelwertbegriffs erarbeitet werden.

\section{Von der Definition zum Satz}

Eher traditionelle Schulbücher präsentieren den zu erlernenden Begriff direkt - zumeist hervorgehoben in einem roten Kasten:

1. Definition: $\quad x$ heißt ... (Begriffswort) $: \Leftrightarrow$ Aussage $_{\text {def }}$ über $x$

2. Satz: $\quad$ Aussage $_{\text {def }}$ über $\mathrm{x} \Leftrightarrow$ Aussage $_{\text {satz }}$ über $\mathrm{x}$

Hierbei muss angemerkt sein, dass statt der Äquivalenz, die hier zwischen der definitorischen Aussage (Aussage def $_{\text {) }}$ und der Aussage des Satzes (Aussage $_{\text {Satz }}$ ) vermittelt, lediglich eine konditionale Folgerung vorliegen kann.

Am Beispiel des arithmetischen Mittelwertes stellt sich diese Struktur wie folgt dar:

1. Definition: $\quad x$ heißt arithmetischer Mittelwert $: \Leftrightarrow\left(a_{1}+\ldots+a_{n}\right): n=x$

2. Satz: $\quad\left(a_{1}+\ldots+a_{n}\right): n=x \Leftrightarrow\left(a_{1}-x\right)+\ldots+\left(a_{n}-x\right)=0$

\section{Begriffsbildung durch Entdecken und Begründen am Beispiel des Mittelwertes}

In neueren Schulbüchern ist es eher üblich, dass vor der expliziten Thematisierung eines Begriffs einführende Aufgaben gestellt werden. Diese Aufgaben können dazu dienen, dass die Lernenden ihr bisheriges Wissen von einem ihnen vertrauten Kontext aufrufen, in dem der zu erlernende Begriff teilweise impliziert ist. Beispielsweise wird der Begriff Mittelwert in reduzierter Bedeutung als „mittlere Zahl“ einer Zahlreihe erfahren:

$5+6+7=\square: \square: 3=$

An dieser und ev. weiteren Summen von drei aufeinanderfolgenden Zahlen kann entdeckt werden, dass sich bei der Division der Summe durch 3 stets die „mittlere Zahl“ ergibt. Die Begründung, dass diese Regel immer gelte, kann durch das gegensinnige Verändern von zwei Summanden erfolgen:

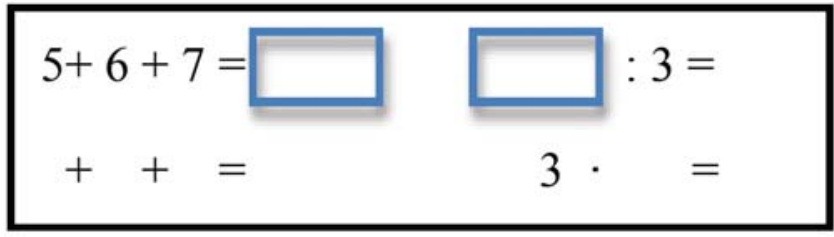

Abb.: Eine Startaufgabe zum Begriff ,arithmetisches Mittel“ 
In der ersten Zeile der Abb. wird die Definition des Mittelwertes in reduzierter Weise konkretisiert. Das gegensinnige Verändern erfolgt auf der linken Seite von oben nach unten. Wenn man weiter entgegen dem Uhrzeigersinn über die fortgesetzte Addition als Multiplikation und über die Umkehrung der Multiplikation zur Division voranschreitet, ergibt sich die Begründung. Zur Begründung der allgemeinen Regel ist von den Beispielzahlen zu abstrahieren auf beliebige Summen von drei aufeinander folgende Zahlen (s. beispielgebundenes Begründen, Krummsdorf 2009). Damit wird der obige Satz in der Richtung „Æ“ begründet, eingeschränkt auf drei aufeinander folgende Zahlen. Die Begründung der Richtung „ $\Rightarrow$ “ kann entsprechend im Uhrzeigersinn erfolgen.

Um dem Allgemeinheitscharakter des Mittelwertes gerecht zu werden, wird nun die Anzahl der Summanden variiert, die Abstände zwischen den Summanden werden vergrößert und auch Zahlen in nicht-äquidistanten Abständen werden vorgegeben. Diese Aufgaben lassen sich auch in alltagsbezogenen statistischen Kontexten präsentieren, was hier aus Gründen des Umfanges unterlassen wird. Wenn in dieser Folge Summen wie 1+2+7+30 zu bearbeiten sind, so ist der Weg über das gegensinnige Verändern entgegen dem Uhrzeigersinn jedoch mühsam. Vielmehr gibt dann der Weg über die definitorische Aussage den Anlass, 10 nicht nur als Ergebnis der Division „(1+2+7+30):4“ festzustellen, sondern auch zu vermuten, dass 10 durch gegensinniges Verändern erzielt werden kann. Dies kann durch $(1+9)+(2+8)+(7+3)+(30-20)$ bestätigt werden, was wiederum Anlass zu der Entdeckung gibt, dass bei beliebigen Summen der entsprechende Quotient gleich einer durch gegensinniges Verändern erzielbaren Zahl ist, analog zur früheren einfacheren Situation bei drei aufeinander folgenden Zahlen. Die Begründung erfolgt beispielgebunden oder (in höheren Klassenstufen) formalisiert in algebraischer Sprache. Beim Übergang von der einfacheren Situation 5+6+7 über 8+10+9, 11+13+15+17+19 etc. bis zu Summen ohne Regelmäßigkeit verändert sich der Begriff „mittlere Zahl einer Zahlreihe“ zum Begriff „arithmetischer Mittelwert“. Auf diese Weise haben die Lernenden die Gelegenheit, zwei gleichwertige „Definitionen“ des arithmetischen Mittelwertes zu entdecken und ihre Gleichwertigkeit zu begründen. Anschließend besteht dann immer noch die Möglichkeit, den neuen, veränderten Begriff zu „taufen“, d.h. mit der offiziellen Bezeichnung „Mittelwert" zu versehen.

Die Kleinschrittigkeit der obigen Aufgabenfolge bei der Begriffsbildung dient dazu, die logischen Schritte des Entdeckens und Begründens herauszuarbeiten, so dass man auch bei offeneren, ganzheitlichen Lernumgebungen wünschenswerte Wege der Lernenden jenseits unreflektierter Verall- 
gemeinerungen vor Augen hat und insbesondere stärker das Begründen in den Fokus der Aufmerksamkeit rückt.

\section{Logische Struktur des Begriffsbildens durch Entdecken und Be- gründen}

Hier soll nicht(!) behauptet werden, die beschriebene Bildung des Begriffs „arithmetischer Mittelwert“ sei anderen Wegen vorzuziehen, obwohl sich dieser Weg in den Klassen 3 und 4 (Grundschule) als gangbar erwiesen hat. Vielmehr dient das Beispiel zur Konkretisierung einer allgemeinen Strategie, mittels der ein Begriff durch Entdecken und Begründen gebildet werden kann.

Die allgemeine Darstellung dieser Strategie erfordert den Einsatz logischer Begriff, insbesondere der Verwendung der Schlussformen „Abduktion“ (für das Entdecken) und „Deduktion“ (für das Begründen) (vgl. Meyer 2007). Während die logische Analyse im Vortrag ausgeführt werden konnte, muss hier aus Gründen des Umfanges auf künftige Veröffentlichungen verwiesen werden. Es sei nur angemerkt, dass die Strategie auch für weitere Begriffe in Form von Folgen von Entdeckungs- und Begründungsaufgaben ausgearbeitet wurde, zum Beispiel zum Bruchzahlbegriff und zum begriff der Mittelsenkrechten.

\section{Literatur}

Fischer, R. \& Malle, G. (1985): Mensch und Mathematik. Mannheim: Bibliogr. Inst.

Götz, S. \& Siller, H.-S. (2010): Vom Modellieren zum Definieren oder: Mathematik(unterricht) rund ums Ei. In: Beiträge zum Mathematikunterricht.

Krumsdorf, J. (2009): Beweisen am Beispiel. Beispielgebundenes Begründen zwischen induktivem Prüfen und formalem Beweisen. In: Praxis der Mathematik in der Schule, 51(39), S. 8-13.

Meyer, M. (2007): Entdecken und Begründen im Mathematikunterricht. Von der Abduktion zum Argument. Hildesheim: Franzbecker.

Rényi, A. (1967): Dialoge über Mathematik. Basel: Birkhäuser.

Vollrath, H.-J. (2001): Grundlagen des Mathematikunterrichts in der Sekundarstufe. Heidelberg: Spektrum.

Winter, H. (1983): Über die Entfaltung begrifflichen Denkens im Mathematikunterricht. Journal für Mathematik-Didaktik, 3, 175-204. 